\title{
Preferencias alimentarias en los hogares de la ciudad de Medellín, Colombia
}

\section{Food preferences in the homes of Medellin city, Colombia}

Luz Marina Arboleda Montoya

Universidad de Antioquia. Medellín, Colombia.

E-mail: luzmar.arboledaœgmail.com

Paula Andrea Villa Alcaraz

Colegiatura Colombiana Institución Universitaria. Medellín, Colombia.

E-mail: paula.villaœcolegiatura.edu.co

\section{Correspondencia}

Luz Marina Arboleda Montoya

Calle 70 No. $52-21$, Apartado Aéreo 1226, Medellín, Colombia.

\begin{abstract}
Resumen
Las preferencias y rechazos de alimentos se constituyen en factores que determinan la elección, las prácticas de consumo, de comercialización, y fundamentalmente el estado de nutrición y salud de los habitantes de la ciudad. Este artículo se propone aportar al interpretar las tendencias de consumo y preferencias alimentarias en los hogares de Medelín. Empleó el método mixto (cuantitativo y cualitativo) con utilización de técnicas como encuestas, entrevistas, grupos focales y talleres. Los resultados muestran que las preferencias alimentarias están determinadas por procesos de representación y significación, con influencias marcadas de la tradición cultural regional. En este sentido, existe una prevalencia en el consumo de las tres ingestas principales (especialmente el almuerzo), así como de alimentos que corresponden a la costumbre y que se entienden de carácter identitario como fríjol, arepa, arroz y carne. Los sabores preferentes corresponden también a la costumbre cultural, como son el salado y el dulce. Además, se evidencia el interés por alimentos considerados más saludables o nutritivos. Palabras clave: Medellín; Cultura Alimentaria; Gusto; Preferencias Alimentarias; Consumo de Alimentos.
\end{abstract}




\section{Abstract}

Food practices are issues that determine the choice, consumption practices, marketing, and fundamentally, nutritional and health status of the inhabitants of the city. This article aims, specifically, the analysis of consumer trends and interpreting food preferences of households in the city of Medellin. The research employed mixed methods for gathering information, using techniques such as surveys, interviews, focus groups, and workshops. Results have identified that food preferences are determined by processes of representation and meaning, with strong influences of regional cultural tradition. In this sense, there is a prevalence in the consumption of the three main intakes (especially lunch) and foods that correspond to custom and identity, as beans, corn bread, rice, and meat. Preferred flavors are also cultural habit, such as salty and sweet. Additionally, interest in food considered healthier or nutritional is identified.

Keywords: Medellin; Food Culture; Taste; Food Preferences; Food Consumption.

\section{Introducción}

La alimentación, como interacción social en la que confluyen -en permanente transformación- las intersubjetividades de individuos y colectividades, mezcla factores relacionados con la producción, la economía, la política, la cultura, la ética, la estética y -principalmente- la supervivencia, por lo que se sitúa en un lugar fundamental dentro de las dinámicas humanas, en tanto es hecho y acción que determina además de lo biológico, diferencias e identidades, así como las condiciones de bienestar y autorrepresentación de los sujetos/comensales, pues según Aguirre (2004a) la alimentación nunca es exclusivamente social ni biológica, sino que en este acto se integran ambos.

Entre los factores determinantes de las prácticas alimentarias están los ingresos económicos, generalmente condicionantes del acceso a una alimentación apropiada y suficiente. Un asunto relacionado con las inequidades que se viven en esta región latinoamericana, donde el 10\% de la población ostenta el 70\% de los recursos, y la pobreza está extendida hasta tal punto que el $20 \%$ de las personas vive con menos de dos (2) dólares al día (OPS, 2012), lo que propicia las dificultades que atraviesan las poblaciones en relación con acceso a la comida.

Así mismo, las elecciones que, en atención a sus preferencias, realizan los encargados de la alimentación doméstica y el comensal mismo, están usualmente permeadas por factores de tipo cultural, que depositan significados sobre los alimentos, las preparaciones y las experiencias que proporcionan; al establecer signos, simbolismos que dan paso a las costumbres y a los hábitos alimentarios (Gracia, 1996). Otro factor que influye en las preferencias y por tanto en la elección alimentaria, especialmente en el ámbito contemporáneo, es lo que se deriva de los lineamientos de la nutrición, y que han hecho que se incremente el interés por la salud del cuerpo y la mente. A esta intervención se suman las marcadas por la publicidad de alimentos (cada vez en aumento), otro elemento que influye en las formas de comer de individuos y grupos poblacionales (Aguirre, 2004b).

De acuerdo a lo anterior, en la alimentación se integran factores tanto socioculturales como eco- 
nómicos y nutricionales - de orden tradicional y moderno -, que conforman una hibridez que da como resultado unas maneras particulares de alimentación en los diversos territorios. Al respecto García (2001) plantea que las mezclas culturales y sociales entre lo tradicional y lo moderno se constituyen en la incertidumbre de la modernidad.

En este contexto, las prácticas de la alimentación se modifican velozmente. Las nuevas tendencias alimentarias traen consigo cambios en aspectos simbólicos, estéticos y nutricionales, que repercuten en las formas de comer, los tipos de alimentos consumidos, en el estatus y usos dados a los alimentos. Muchas de estas percepciones y significados, que los diferentes grupos poblacionales atribuyen al acto de alimentarse, son dados por las dinámicas establecidas en el ámbito social y familiar, especialmente en su intento de acceder a los alimentos y preservar la alimentación acorde a los hábitos alimentarios aprendidos, que en ocasiones trae como consecuencias estados nutricionales precarios y hambre oculta (Aguirre, 2004b).

La sociedad contemporánea tiene múltiples contrastes en lo referente al consumo de alimentos. Por un lado, las desigualdades sociales marcan fuertemente los accesos y las preferencias de consumo, puesto que la selección de alimentos dependen del ingreso de la familia, la cual se ve en la obligación de elegir alimentos baratos y saciadores, que van forjando el gusto por unos grupos de alimentos. Por otro lado, y no siendo el único contraste, se encuentran grupos preocupados por consumir alimentos benéficos para su salud y su cuerpo (Fischler, 2005), así como otros - la mayoría - que consumen alimentos por practicidad, sin importar su contenido nutricional ni sus repercusiones para la salud.

Para apoyar las necesidades sociales, la industria de alimentos desarrolla productos de consumo que cumplen con estas características, además, realiza un gran despliegue de campañas publicitarias y estrategias de marketing que, en la mayoría de los casos, solo buscan aumentar las utilidades y alentar un sistema económico basado en el consumismo (Díaz; Gómez, 20o8).

Así la población se enfrenta a las demandas y a la seducción de la globalización y de los medios masivos de comunicación, que incitan al consumo de alimentos procesados, con altas cantidades de grasas saturadas, trans, sodio y carbohidratos, y a situaciones de pobreza y hambre, lo cual trae como consecuencias desnutrición, obesidad y presencia de enfermedades crónicas no transmisibles como la diabetes y dislipidemias, entre otras.

Dichas tendencias alimentarias, diferenciadas por factores sociales y culturales, conllevan a la malnutrición de gran parte de la población, tanto por déficit como por exceso, y en especial este último. La Encuesta Nacional de la Situación Nutricional 2010 (ICBF, 2010) evidencia dicha situación, con prevalencias de sobrepeso u obesidad en la población en general de $52 \%$, y de grupos específicos como el de 5 a 17 años de $17,5 \%$.

El consumo de alimentos en lo urbano está permeado por las influencias de los medios de comunicación, la publicidad, la salud y la nutrición, disponibilidad y las dinámicas de acceso a los alimentos, la ética y las estéticas y los intercambios de la globalización; aspectos paralelos a la cultura, en tanto costumbre, tradición y expresión viva de lo humano, aspectos que determinan el comer en lo cotidiano y en ocasiones especiales.

En este artículo se pretende describir las preparaciones consumidas en los hogares de Medellín e interpretar las preferencias alimentarias relacionadas con el gusto y el sabor.

\section{Metodología}

Esta investigación se realizó en hogares de los diferentes estratos socioeconómicos de la ciudad de Medellín. Se orientó desde los enfoques cualitativo y cuantitativo debido a que el objeto de estudio elegido, la alimentación, es complejo. Cada uno de los enfoques aportó a la comprensión de dicho fenómeno y brindó gran comprensión teórica para el análisis de la información (Himmelgreen; Crooks, 2005).

El enfoque cuantitativo se utilizó con fines exploratorios, entendiendo su aplicabilidad cuando el objetivo es examinar un tema poco estudiado (Sampieri; Fernández; Baptista, 1991), y comprendiendo que en la ciudad de Medellín poco se ha indagado por las prácticas y significados de la alimentación. 
Para llevar a cabo la recolección de los datos, se diseñó un cuestionario con preguntas abiertas en su mayoría, con el fin de indagar por aspectos correspondientes a prácticas y pautas alimentarias en los hogares de los diferentes estratos. Se tomó una muestra, aleatoria estratificada, de 384 hogares de la zona urbana de la ciudad de Medellín, que fue suficiente para describir la composición de las comidas en los diferentes momentos del día. La prevalencia del fenómeno (prácticas y significados de la alimentación) se asumió en un 50\%, pues no se han encontrado estudios previos similares en esta población. La muestra se calculó con base en los siguientes parámetros: confianza - 95\%, población - 577.876 hogares según la encuesta de Calidad de Vida (2008) y error de muestreo $-5.0 \%$.

El enfoque cualitativo permitió interpretar las elecciones y gustos alimentarios en los hogares al privilegiar "lo local, lo cotidiano y lo cultural", e identificar las realidades alimentarias, la diversidad y la riqueza de significados atribuidos a este fenómeno (Minayo, 2003, p. 12).

El método empleado fue la etnografía que describe las sociedades humanas, como base del conocimiento que pretende la antropología (Vallés, 1997). En este caso, los grupos de personas del estudio compartían las dinámicas sociales, económicas y culturales.

Se utilizaron tres técnicas de recolección de información: grupos focales, entrevistas individuales y talleres. Para desarrollar dichas técnicas se diseñó un guion de entrevista, el cual orientó las conversaciones alrededor de las temáticas propuestas.

Con los grupos focales se buscó que las personas discutieran y elaboraran, desde la experiencia personal, aspectos de la alimentación (Morgan; Krueger, 1998). En total se realizaron seis grupos focales, en los que participaron mujeres, en su mayoría amas de casa, de diferentes estratos socioeconómicos y barrios de la ciudad como San Javier, Laureles, Belén, Castilla, Doce de Octubre y La América.

La entrevista abierta (semidirectiva) se establece como un proceso de producción intercomunicativo, por el cual un interlocutor produce información en un contexto social determinado (Valero, 2005).
En total se efectuaron veinticuatro entrevistas, en su mayoría a mujeres, residentes en las distintas comunas de la ciudad y de los seis estratos socioeconómicos.

Finalmente, el taller se entendió como un espacio de aprendizaje en el que todos los participantes discuten acerca de temas específicos o de interés para la realización de un proyecto (Grudndmann; Stahl, 2002). En total se realizaron seis talleres en diferentes barrios de Medellín, con personas de los diversos estratos socioeconómicos.

Respecto a la alta participación de mujeres en las diversas técnicas de la investigación, se consideró que estaba acorde a la realidad y cotidianidad de los hogares de la ciudad. En todo el país, las mujeres son quienes están encargadas de la alimentación en los hogares, son quienes, cada vez más, acceden a los alimentos, los eligen, compran, preparan y distribuyen; además, por ser quienes conocen los gustos de los miembros de las familias, realizan las compras y preparaciones de los alimentos en función de dichos gustos.

\section{Consideraciones éticas}

Para desarrollar las técnicas cualitativas se invitó a los sujetos a que participaran de manera voluntaria, estableciéndose relaciones horizontales. Así mismo, se les pidió que firmaran un consentimiento informado en el cual se les indicaba el objetivo de la investigación, la manera en que se desarrollaría la técnica y la duración de la misma, que la conversación sería grabada. Se les informó, además, acerca del procesamiento de la información, en el cual se les aseguró el anonimato a través de la sustitución de sus nombres por códigos, se aclaró que la información generada solo se utilizaría con fines académicos, y - por último - se les garantizó que la información generada estaría guiada por la veracidad (Vallés, 1997; Galeano, 2004).

\section{Procesamiento de la información}

Los datos, obtenidos con los métodos cuantitativos, se describieron por medio de porcentajes y la prueba de diferencia de proporciones. Para el 
procesamiento de la base de datos y el análisis de la información se utilizaron el SPSS y el Excel.

Adicionalmente se procedió a identificar las proporciones de los datos en forma general y por estrato, utilizando a la vez información cualitativa, la cual proporcionó explicaciones y significados a las tendencias descritas en los datos numéricos.

Para el procesamiento de la información cualitativa obtenida se recurrió al diseño de una matriz en Excel, estableciéndose en el eje de las columnas aspectos como: técnica, lugar, fecha, participante (s), estrato, temáticas (pautas, prácticas, creencias, significados); luego se procedió a triangular los datos obtenidos por diferentes técnicas y fuentes bibliográficas, lo que permitió la identificación y comprensión de dos categorías, analizadas en forma general para Medellín y por estrato socioeconómico, así:

- Consumo alimentario en hogares de Medellín;

- Preferencias alimentarias en Medellín.

\section{Resultados}

\section{Consumo alimentario en hogares de Medellín}

Debido a la diversidad social, económica y cultural de la población de Medellín se evidencian cuatro tendencias de consumo alimentario: tradicional, práctica-moderna, saludable y de escasez. Cada tendencia se identifica por el tipo de alimentos consumidos, por las preferencias alimentarias, por la calidad de los alimentos consumidos y por el lugar de consumo de las comidas, entre otras.

En términos generales, en los hogares de Medellín se consumen tres comidas principales y tres o cuatro entrecomidas, que representan el arraigo de una práctica rural histórica, que migró a la ciudad. Estos momentos de comida son: tragos, desayuno, mediamañana, almuerzo, algo, comida y merienda.

Los tragos son un momento de comida instaurado en la cultura paisa que todavía es conservado por la mitad de los hogares de Medellín, especialmente en los de estratos más bajos. En dichos hogares se consume el tinto (café) y el café con leche como práctica tradicional, también se identifican consumos diferenciados por estrato como el agua y el jugo de naranja, en los altos, lo cual tiene una connotación de bebidas nutritivas y saludables. Este momento de comida se modifica en la actualidad por la influencia de las percepciones y la reconfiguración, especialmente en los estratos con mejores condiciones socioeconómicas, que transforman las representaciones de la costumbre y lo relacional, para otorgarle características ligadas a la salud y la nutrición.

Con respecto al desayuno, ligado a lo tradicional, se prefiere principalmente alimentos como: arepa con quesito o queso, o arepa con huevo y chocolate. Asímismo, se evidencia, en menor proporción (13\%), los consumos de solo cereal como arepa o pan o galletas o tostadas en hogares ubicados en estratos bajos, considerada como una tendencia de escasez. Otra tendencia en el consumo al desayuno es la de cereales con leche o yogurt en hogares de estratos altos, como tendencia moderna-práctica.

La principal bebida ingerida en los hogares al desayuno, sin distinción de estrato, es el chocolate, seguida del café con leche; además del consumo de otras dos bebidas que sí guardan relación con el estrato socioeconómico, como la aguapanela consumida en los estratos más bajos y la gaseosa en los más altos. La mediamañana es consumida en un $35,7 \%$ de los hogares, especialmente en los estratos más altos, a esa hora se consumen principalmente frutas enteras o en jugo, y en menor proporción café (tinto).

El almuerzo es el momento de comida en el cual se come de forma más estructurada y en mayor abundancia. Dicho consumo se da en tres platos: sopa (entrada), seco (plato fuerte) y sobremesa (bebida). Las sopas son preparadas de fríjoles, legumbres, pastas o papa y yuca. El seco está compuesto de arroz, tajadas de plátano maduro o de papa, ensalada y carne de res, o de cerdo o pollo. También se presentan consumos como los anteriores, pero sin ensalada o sin tajadas, y los consumos de solo cereales o tubérculos o plátanos, como el consumo de solo arroz o solo sopa, los cuales connotan consumos de escasez que conllevan al hambre oculta. De sobremesa toman principalmente jugo de fruta, y en menor proporción leche, refresco en polvo o aguapanela. 
El algo es un momento de comida presente en el $46,9 \%$ de los hogares de la ciudad, es una práctica más realizada en hogares de estratos altos. A esa hora del día en los hogares consumen principalmente: café con leche y pan (en ocasiones galletas) o tinto o frutas.

La comida (cena) es el momento del día que más cambios ha presentado respecto a la composición y cantidad de alimentos y preparaciones. Esta sigue dos tendencias, una tradicional, compuesta por sopa y seco, muy parecida a la presente en la hora del almuerzo, pero en menor cantidad, y una tendencia más ligera y moderna ligada a factores de salud, cuya composición es muy parecida a la del desayuno, conformada por arepa acompañada con carne o jamón o queso y bebida fría o caliente. A la hora de la comida también se presenta consumo de escasez, es decir, ingesta solo cereales como arroz o arepa.

Y el último momento del día es el menos utilizado, la merienda es consumida solo en el 19\% de los hogares sin presentar diferencias por estrato socioeconómico. A esa hora se consume café con leche con pan o galletas y, en menos proporción, chocolate con leche y un cereal, o simplemente agua.

\section{Preferencias alimentarias en Medellín}

Frente a las referencias alimentarias en relación con el sabor en Medellín, la investigación requirió comprender, en una instancia previa -que se verificó en los procesos de pilotaje-, las maneras particulares de denominación y clasificación del sabor para la región. Así dulce, amargo, salado, agrio, picante (códigos comunes y reconocidos por el paladar occidental), umami, acre (categorizaciones heredadas de la experiencia de comensalía de oriente) y el sabor adiposo (propuesto de manera reciente por investigadores de la Universidad de Bolonia), fueron remplazados en el parámetro desarrollado para las encuestas por dulce, amargo, salado, ácido, picante, ahumado y simple, sabores reconocidos por los medellinenses para clasificar la experiencia sensorial del gusto.

Entre los hallazgos principales, la experiencia dulce se instala como preferencia predominante en los hogares encuestados, quienes en un $62 \%$ la establecen como su favorita. La preferencia de este sabor en el estrato 1 está presente en un 59\% de los hogares, prevalencia que se mantiene en los estratos 2, 3, 4 y 5, pues en el 6 se evidencia una mayor preferencia por el sabor salado $(60 \%)$, mientras que por el dulce un $47 \%$.

El sabor salado en la ciudad presenta una preferencia del $44 \%$ verificable en relación con el consumo de alimentos cotidianos y extraordinarios, y se constituye en la segunda predilección.

Ahumado y simple, experiencias del gusto que no corresponden con las clasificaciones reconocidas, son entendidas por los comensales de Medellín como experiencias de sabor comunes y frecuentes, especialmente el simple, con una prevalencia que se ubica en el tercer lugar entre las familias encuestadas, con un $21 \%$.

En relación con los sabores agridulce (15\%) y picante (14\%) es posible identificar la importancia de las mezclas de sabor en el primer caso, percepciones que responden a prácticas culinarias relacionadas con las celebraciones (generalmente salsas y aderezos) y a recetas adaptadas de otras culturas, que como en el caso de la comida china, se consumen con frecuencia en ocasiones especiales familiares. En relación con el picante, es posible reconocer la importancia de este sabor -vestigio de la tradición indígena-y de su preferencia como acompañamiento en ajíes principalmente.

Finalmente, el sabor amargo, con una escasa prevalencia del $2 \%$, pese a la importancia del consumo de bebidas tradicionales como el café que corresponden a dicha experiencia, tiene una mínima preferencia entre los comensales, que poco reconocen este sabor como el más apetecido.

Dejando de lado las prevalencias relacionadas con los sabores, es importante enunciar las preferencias correspondientes al consumo de alimentos, especialmente preparados. Un hallazgo significativo es la preferencia por el consumo de fríjoles (en sopa) y carne en un 32,3 y $22,1 \%$ respectivamente, más significativa en la percepción de los estratos 1, 2 y 3 . El consumo de frijoles y carnes responde a algunas de las prácticas más representativas que se conservan de la tradición culinaria antioqueña, sin embargo, esta preferencia se modifica ligeramente en las prácticas de alimentación de los demás 
estratos socioeconómicos, en los cuales aparecen adicionalmente como favoritos otros productos $y$ preparaciones como las frutas (estrato 4), las ensaladas (estrato 5) y los camarones y mariscos (estrato 6); estos últimos significativamente distantes de la cocina tradicional de la región.

Es importante también destacar la preferencia por el consumo de pollo, en un $17,4 \%$, y el de arroz (tanto solo como chino y con pollo), elegido por gusto principalmente $(60,7 \%)$ y por costumbre $(31,1 \%)$; también es significativa la predilección por las sopas $(15,9 \%)$ y el sancocho $(15,6 \%)$, preparaciones del orden tradicional.

En lo que respecta a los rechazos de alimentos, se evidenció que las lentejas y garbanzos, generalmente utilizados en sopas, son rechazados en un $27,1 \%$. Paradójicamente, también se registran rechazos significativos por las sopas (14,3\%), el pescado y la sardina, en un $9,4 \%$. Lentejas y garbanzos son rechazados principalmente en los estratos 1, 2, 3 y 5 , en el estrato 4 el pescado y en el estrato 6 las menudencias, los embutidos y el mondongo.

\section{Discusión}

El consumo y las prácticas alimentarias que llevan a cabo los diferentes grupos y sociedades son de origen complejo por la incidencia e interacción de factores sociales, culturales y económicos, entre otros, derivando además comportamientos que obedecen a la tensión que se da entre las prácticas, consumos y alimentos asociados con lo tradicional y con lo moderno (Díaz; Gómez, 20o8). Los nuevos alimentos disponibles, ligados a lo moderno e industrializados, contienen gran cantidad de aditivos, saborizantes, colorantes, preservantes y nutrientes trazadores de riesgo para la salud como azúcares añadidos, sodio y grasas trans, entre otros (Arboleda; Velásquez; Varela, 2014), y - a pesar de esto - tienen el privilegio de ser promocionados por diferentes medios masivos de comunicación. Por su parte, los alimentos ligados a lo tradicional se transmiten por medios intra e intergeneracionales (Gracia; Contreras, 2005).

Respecto a lo anterior, en Medellín persisten tendencias de consumo asociadas a lo tradicional, evidenciadas en el gran porcentaje de hogares que aun consumen las comidas principales, en la comensalía y comunión que permanece en los momentos de la comida, y en los alimentos y preparaciones de mayor preferencia en los hogares como los fríjoles, el arroz y la arepa, lo cual es identificado en una investigación realizada en la ciudad. (Arboleda; Ochoa, 2013). Sin embargo, también existen unas prácticas ligadas a la modernidad, poco extendidas, como las prácticas del picoteo, el consumo de alimentos industrializados y poco nutritivos como las gaseosas.

Con lo anterior, se resalta la importancia de una categoría cultural como es el sabor de los alimentos, porque configura unos gustos particulares en cada grupo y sociedad. En la ciudad de Medellín, por ejemplo, se tiene preferencia por un sabor inexistente como es el simple, percepción posiblemente ligada al consumo de unos alimentos tradicionales como la arepa, cuyo sabor es insípido, o indicadora de un interés por atender las demandas ligadas a la nutrición y a la salud. Por el mismo motivo, las prácticas relacionadas con el consumo de alimentos de sabor dulce no son declaradas por la población, o, por el contrario, son alimentos que ya no hacen parte de las prácticas y dieta cotidianas.

La familiaridad que se tenga con los alimentos se constituye en un factor que influye en el gusto de los sujetos por algunos de ellos, por consiguiente, en los hogares de menor acceso a alimentos, las preferencias por otros sabores diferentes al simple, salado y dulce son limitadas y, así, la posibilidad de elegir por gusto y preferencia otros sabores característicos como el de las frutas y las verduras es también limitado. A lo anterior se suma la connotación que tienen las frutas y las verduras de alimentos poco saciadores (Aguirre, 2004b), lo cual hace que este grupo de alimentos sea poco preferido y elegido, tal como se encontró en los resultados de la investigación realizada en la región de Urabá, Colombia, donde las familias prefieren comprar alimentos como tubérculos, cereales y plátanos por su gran poder de saciedad, en vez de frutas (Arboleda; Duque; Urrea, 2013).

De esta manera, el factor económico y la clase social configuran diferencias en la percepción que los grupos poblacionales tienen de este grupo de 
frutas y verduras, instaurando preferencias vinculadas a la importancia que cobra, en las dinámicas actuales de la alimentación, la relación con la salud y nutrición como una tendencia de modernidad alimentaria, constituyéndose en un factor de elección que se alterna con el gusto individual y las costumbres culturales. En todos los estratos de la ciudad, las frutas tienen una connotación de alimento saludable y nutritivo, no obstante, en los estratos más bajos estos alimentos también tienen un significado de alimento costoso y de lujo, a los que solo pueden acceder cuando tienen un poco más de dinero o cuando están enfermos; mientras que en los estratos altos este es un gusto de distinción al que pueden acceder sin tener que pensar si les brindará saciedad o no.

A nivel de país también se han identificado tendencias alimentarias tradicionales con algunos rasgos de hibridez. Lo anterior se puede evidenciar en la frecuencia del consumo de ciertos alimentos y preparaciones, por ejemplo, la alta frecuencia (76\%) en el consumo semanal en los hogares de granos o leguminosas, como los fríjoles. La alta proporción de sujetos que nunca consumen comidas rápidas (50\%) ni alimentación por fuera del hogar $(60,6 \%)$. No obstante, dichas prácticas presentaron frecuencias semanales en un 24,5 y $25,3 \%$, respectivamente (ICBF, 2010), de esta manera, se identifican unos consumos híbridos, que unen prácticas de ambas tendencias, tradicionales y modernas.

En Cuba, por ejemplo, los patrones de consumo alimentario en mayores de 15 años son homogéneos en todo el territorio, pues se conserva una mayor tendencia a la alimentación tradicional y se presentan en menor medida las características de una alimentación saludable y nutritiva por el bajo consumo de frutas y verduras y el alto consumo de azúcares, dulces y cereales (Porratas-Maury, 2008). Respecto a las prácticas alimentarias en adolescentes cubanos, el patrón de consumo también presenta una tendencia híbrida, es decir, tienen una alto consumo de alimentos ligados a la modernidad como los refrescos y alto consumo de alimentos fuera del hogar, y algunas prácticas que se alejan de lo que representa una alimentación nutritiva y saludable (como tendencia modernizante) como es el elevado consumo de helados, azúcares, grasas saturadas, jamones y embutidos (Macías et al., 2009) y, a la vez, un elevado consumo de dulces, fríjoles y condimentos naturales (cebolla, ajo, tomate y ají) como prácticas tradicionales.

\section{Conclusiones}

En la ciudad de Medellín se observa una tendencia preferente por alimentos tradicionales, sin importar el estrato socioeconómico, identificado en el consumo de productos de arraigo cultural como el chocolate, el tinto y el café con leche, la arepa, los fríjoles y el arroz. A la influencia de la tradición se mezclan otros aspectos tales como la exposición o el conocimiento de nuevos productos, factores relacionados con el ingreso económico que incide en el acceso a los alimentos y en el gusto alimentario.

Las comidas principales se instauran como comunes en las preferencias alimentarias de los hogares de la ciudad de Medellín, entendiéndose como comidas fundamentales, especialmente el almuerzo. Las demás ingestas se consideran como complementarias, entre las que se destacan prácticas en transformación como las referidas a los tragos, mediamañana, algo y merienda. Del mismo modo, la composición de las comidas presenta diferentes tendencias tradicionales, modernas y de escasez. Esta última evidenciada en el consumo, en las principales comidas, de platos compuestos de preparaciones a base de cereales o tubérculos como arepa, arroz o sopas.

En la ciudad de Medellín se presenta una gran preferencia por alimentos dulces y salados, así como el rechazo general por los alimentos amargos. En relación con la predilección por el sabor dulce, común a todos los estratos, no se evidencia una correspondencia con la prevalencia en el consumo de preparaciones dulces. La percepción y la preferencia sobre los demás sabores están ligadas a la condición socioeconómica. En los estratos más bajos se identifica una preferencia por lo que denominan como sabor "simple", mientras que en los más altos se consumen y eligen por predilección alimentos con sabores como el picante, el agridulce, el ácido y el ahumado. 
Se pudo identificar que las elecciones alimentarias, en Medellín, están ligadas a factores culturales más que a factores de salud y nutrición. Las preferencias alimentarias están dadas por el gusto, una experiencia que puede ser positiva o negativa frente a los alimentos y que combina sabor, agrado y en ocasiones, costumbre.

La preferencia por la tradición culinaria se evidencia en la importancia que se le da al consumo de fríjol, carne, arroz y un amasijo como la arepa; cuyas recetas han estado inmersas en la historia cultural de la ciudad y la región, estableciéndose los consumos del orden identitario y reconocidos por propios y extraños como referentes estéticos en el ámbito de la comida y el comer.

\section{Referencias}

AGUIRRE, P. Ricos flacos y gordos pobres.

Alimentación en crisis. Claves para todos. Buenos

Aires: Capital Intelectual, 2004a.

AGUIRRE, P. Seguridad alimentaria. Una

visión desde la antropología alimentaria.

2004b. Disponible en: <http://www.suteba.org.

ar/download/trabajo-de-investigacin-sobre-

seguridad-alimentaria-13648.pdf >. Acceso el: 7

enero 2014 .

ARBOLEDA, L. M.; OCHOA, A. M. Estrategias de acceso a los alimentos en los hogares de estrato 1, 2 y 3 de la ciudad de Medellín. Revista Facultad Nacional de Salud Pública, Medellín, v. 31, n. 1, p. 58-66, enero 2013.

ARBOLEDA, L.; DUQUE, M.; URREA, J.

Significados del consumo de frutas y hortalizas en dos comunidades de la zona rural del municipio de Turbo del Urabá Antioqueño. Saúde e Sociedade, São Paulo, v. 22, n. 4, p. 1247-1256, 2013.

ARBOLEDA, L., VELÁSQUEZ, J., VARELA, D.

Presencia de nutrientes trazadores de riesgo para la salud en alimentos consumidos por escolares de Medellín. Revista Facultad Nacional De Agronomía, Medellín, v. 67, n. 2, p. 1055-1057, jul. 2014.

DÍAZ, C.; GÓMEZ, C. (Coor.). Alimentación, consumo y salud. Barcelona: Fundación "la Caixa", 2008.
FISCHLER, C. $E l$ (H) Omnívoro: El gusto, la cocina y el cuerpo. Barcelona: Anagrama, 2005.

GALEANO, M. E. Estrategias de investigación social cualitativa. El giro en la mirada. Medellín: La Carreta, 2004.

GARCÍA, N. Culturas híbridas. Estrategias para entrar y salir de la modernidad. Buenos Aires: Paidós, 2001.

GRACIA, M. Antropología de la alimentación. En: MARTÍNEZ HERNÁEZ, A.; CARÓS, J. P. (Ed.). Ensayos de antropología cultural: homenaje a Claudio Esteva-Fabregat. Barcelona: Ariel, 1996.

GRACIA, M.; CONTRERAS, J. Alimentación y diferenciación social. En: Alimentación y cultura. Perspectivas antropológicas. Barcelona: Ariel, 2005 .

GRUDNDMANN, G.; STAHL, J. Como la sal en la sopa: conceptos, métodos y técnicas para profesionalizar el trabajo en las organizaciones de desarrollo. Bogotá: El Búho, 2002.

HIMMELGREEN, D. A.; CROOKS, D. L. Nutritional anthropology and its application to nutritional issues and problems. En: KEDIA, S.; WILLIGEN, J. van (Ed.). Applied Anthropology: domains of application. Westport, CT: Praeger, 2005. p. 149188.

ICBF - INSTITUTO COLOMBIANO DE BIENESTAR FAMILIAR. Encuesta Nacional de la Situación Nutricional ENSIN 2010. Bogotá, 2010.

MACÍAS, C. et al. Hábitos, actitudes y preferencias alimentarias en adolescentes de enseñanza media de La Habana. 2009. Disponible en: <https://dialnet.unirioja.es/servlet/ articulo?codigo=3062221>. Acceso el: 19 jun. 2016.

MINAYO, M. (Org.). Investigación social: teoría, método y creatividad. Buenos Aires: Lugar Editorial, 2003.

MORGAN, D. L.; KRUEGER, R. A. The focus group kit. Thousand Oaks: Sage, 1998.

OPS - ORGANIZACIÓN PANAMERICANA DE LA SALUD. Salud en las Américas. OPS, 2012. Disponible en: <http://www.paho.org/ 
saludenlasamericas/index.php?option=com_cont ent\&view=article\&id $=58 \% 3$ Ahealth-determinantsand-inequalities\&catid $=24 \% 3$ Achapters \& Itemid $=1$ 65\&lang=es>. Acceso el: 20 jun. 2016.

PORRATAS-MAURY, C. Consumo y preferencias alimentarias de la población cubana con $15 y$ más años de edad. 2008. Disponible en: <https:// www.researchgate.net/publication/261914166_ Consumo_y_preferencias_alimentarias_de_la_ poblacion_cubana_con_15_y_mas_anos_de_edad>. Acceso el: 20 jun. 2016.
SAMPIERI, R.; FERNÁNDEZ C.; BAPTISTA, P. Metodología de la investigación. México, DF: McGraw-Hill, 1991. p. 59.

VALERO, A. Usos y perspectivas sociológicas de la entrevista como técnica de investigación social. Saberes Revista de Estudios Jurídicos, Económicos y Sociales, Madrid, v. 3, n. 1, 2005.

VALLÉS, M. Técnicas cualitativas de investigación social. Madrid: Síntesis, 1997.
Contribución de los autores

Estas autoras han contribuido de igual forma a las etapas de preparación de este texto.

Recibido: 02/05/2015

Revisado: 09/06/2016

Aceptado: 07/07/2016 